\title{
Control of the Boost DC-AC Converter by Energy Shaping
}

\author{
Carolina Albea, Francisco Gordillo and Javier Aracil \\ Escuela Superior de Ingenieros \\ Universidad de Sevilla \\ Camino de los Descubrimientos, s/n, 41092 Sevilla, Spain \\ calbea@cartuja.us.es, gordillo@esi.us.es, aracil@esi.us.es
}

\begin{abstract}
In this paper a control strategy for generation of alternating current without using any reference signal is applied to a nonlinear boost dc-ac converter. A Phase-Locked Loop is added to the control law in order to achieve synchronization between the two parts of the circuit. It is also shown that this idea is also valid for synchronization with the network. The resultant control laws are tested by means of simulations.
\end{abstract}

\section{INTRODUCTION}

In electronics converters with ac output the control objective can be seen as the generation of a stable limit cycle with given amplitude and frequency for which voltages and currents present a sinusoidal behavior with pre-specified phase shift. If a control law is able to produce such a limit cycle, the generation of alternating current will be accomplished without the necessity of introducing any time dependent reference signal. The generation of limit cycles for producing self-oscillations has been successfully applied to electro-mechanical systems [1], [2], [3]. Some applications to electronics converters are [4], [5], where a three-phase UPS and a boost converter are controlled using this idea. Concretely, in the boost converter application, a simple boost converter was used to produce oscillating voltage, which is not centered around zero due to the impossibility of achieve negative voltages with this circuit. Therefore, alternating current was not achieved. In this paper, we deal with this problem using a double boost converter [6] and extending the control law proposed in [5] to this case. It is shown that the direct application of this extension does not fulfil the objective due to the lack of synchronization between both parts of the circuit. In order to achieve the synchronization a phase-locked loop (PLL) is added to the previous control law. This idea can be used for synchronization with the electrical network in order to achieve satisfactory power factor. The resultant controllers are checked by means of simulations.

The rest of the paper is organized as follows.c

\section{Generation of Oscillations by Energy SHAPING}

In power electronic converters with ac output the control objective can be seen as the generation of a stable limit cycle with given amplitude and frequency for which voltages and currents present a sinusoidal behavior with pre-specified phase shift. If a control law is able to produce such limit cycle, the generation of alternating current will be accomplished without the necessity of introducing any reference signal. For this, a target system may be defined and by matching its equations and the system equations a control law can be obtained. In order to define the target system consider the following energy-like function

$$
V_{0}\left(x_{1}, x_{2}\right)=\frac{\omega}{4} \Gamma^{2}\left(x_{1}, x_{2}\right),
$$

with $\Gamma\left(x_{1}, x_{2}\right)=\omega^{2}\left(x_{1}-x_{10}\right)^{2}+\left(x_{2}-x_{20}\right)^{2}-\mu$. Parameters $\omega, x_{10}, x_{20}$ and $\mu>0$ should be chosen so that the closed curve $\Gamma=0$ defines the desired behavior. This curve is an ellipse centered at the point $\left(x_{10}, x_{20}\right)$. We can define a dynamical system such that this closed curve is its limit set. This can be reached adopting $V_{0}$ as a Hamiltonian function, and defining the dynamical system (3)-(4)

$$
\left[\begin{array}{c}
\dot{x}_{1} \\
\dot{x}_{2}
\end{array}\right]=\left[\begin{array}{cc}
-k_{a_{1}} & \frac{1}{\Gamma} \\
-\frac{1}{\Gamma} & -k_{a_{2}}
\end{array}\right]\left[\begin{array}{c}
D_{x_{1}} V_{0} \\
D_{x_{2}} V_{0}
\end{array}\right],
$$

resulting

$$
\begin{aligned}
& \dot{x}_{1}=\left(x_{2}-x_{20}\right)-k_{a_{1}} \omega^{2}\left(x_{1}-x_{10}\right) \Gamma \\
& \dot{x}_{2}=-\omega^{2}\left(x_{1}-x_{10}\right)-k_{a_{2}}\left(x_{2}-x_{20}\right) \Gamma
\end{aligned}
$$

Noticing that

$$
\dot{V}_{0}=-\Gamma^{2}\left(k_{a_{1}} \omega^{4}\left(x_{1}-x_{10}\right)^{2}+k_{a_{2}}\left(x_{2}-x_{20}\right)^{2}\right) \leq 0
$$

and using LaSalle invariance principle it can be seen that for all initial conditions except the origin the trajectories of the system tend to the curve $\Gamma=0$.

The behavior of this target system corresponds to the sinusoidal behavior that it is desired of a dc-ac converter. Constants $\omega, x_{10}, x_{20}$ and $\mu$ are design parameters for the frequency bias and amplitude of the desired behavior while $k_{a_{1}}$ and $k_{a_{2}}$ define the speed of the transient response.

\section{Boost DC-AC Converter: Analysis, Control AND RESUlTS}

The boost inverter is specially interesting because it generates an ac output voltage larger than the dc input one. It is made up of two dc-dc converters and a load 
connected differentially across them Fig. 1. Each converter produces a dc-biased sine wave output, $V_{a}$ and $V_{b}$, so that each source generates an unipolar voltage. The circuit implementation is shown in Fig. 2.

Voltages $V_{a}$ and $V_{b}$ should present a phase shift equal to $180^{\circ}$, which maximizes the voltage excursion across the load [6] Fig. 3.

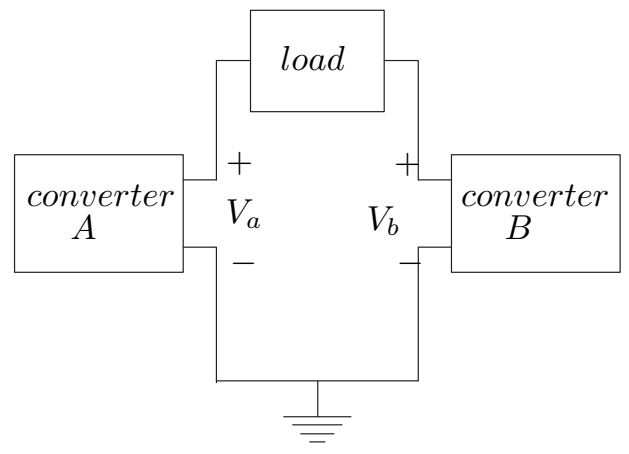

Fig. 1. Basic representation of the boost inverter.

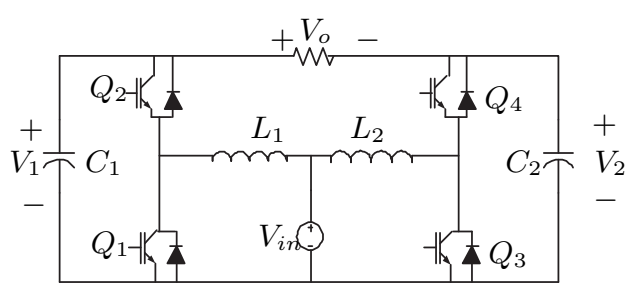

Fig. 2. Ideal Boost dc-ac Converter.
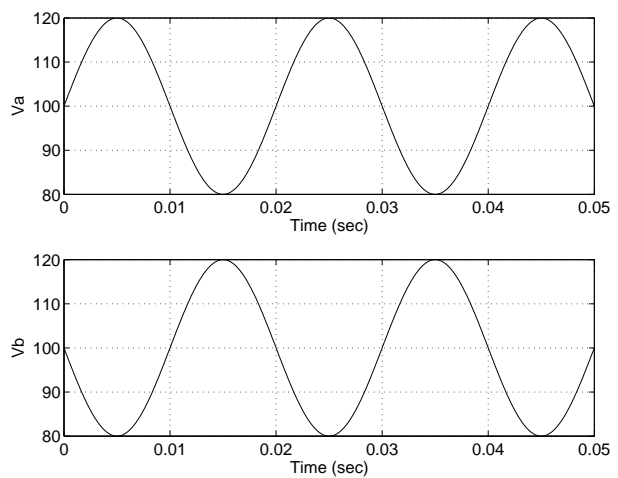

Fig. 3. Ideal output voltages of each boost converter.

The boost dc-ac converter can be simplified as shown in Fig. 4. This simplification lets see clearer the bidirectional current of each boost dc-dc converter.

In order to make easier the studies, we will use this simplification for obtaining the control law, and then we will extrapolate these results to the full converter.

\section{A. System Description}

It is necessary to assume that all the components are ideal and that the currents of the converter are continuous. The two performance modes for a time period are shown in Fig. 5.

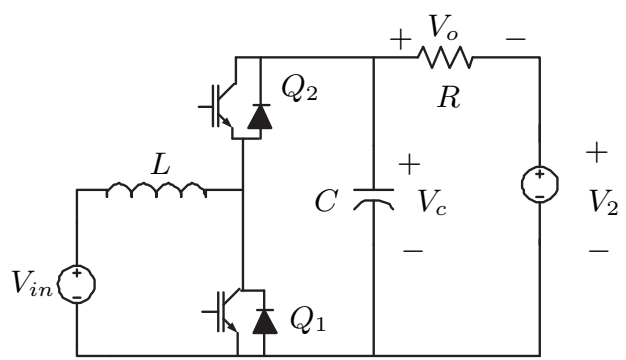

Fig. 4. Symplified Boost dc-ac Converter.
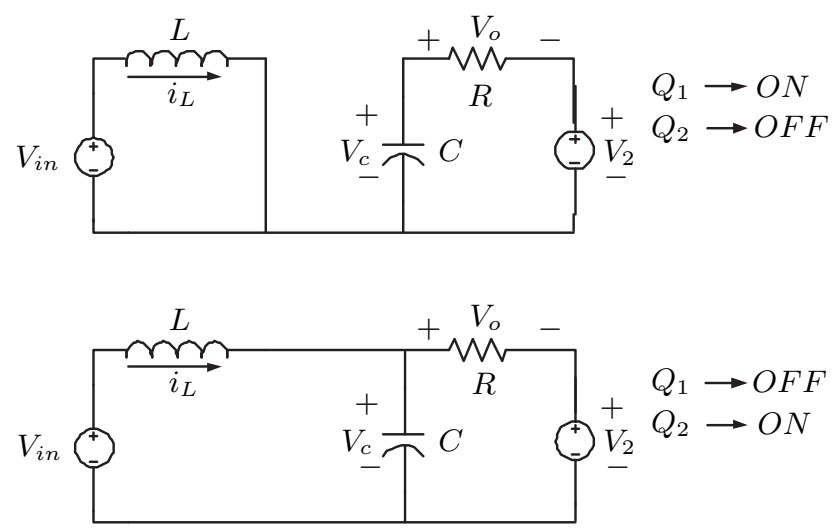

Fig. 5. Operation status.

Defining the control variable $q$ as $q=0$, when $Q_{1}=$ $O F F$ and $Q_{2}=O N$, and $q=1$, when $Q_{1}=O N$ and $Q_{2}=O F F$, the converter dynamic equations are

$$
\begin{aligned}
L \frac{d i_{L}}{d t} & =-v_{C_{1}}+q v_{C_{1}}+V_{i n} \\
C \frac{d v_{C_{1}}}{d t} & =i_{L}-q i_{L}-\frac{v_{C_{1}}}{R}+\frac{V_{2}}{R}
\end{aligned}
$$

If $u=1-q$ is taken as the control action on equations (6)-(7), we have

$$
\begin{aligned}
L \frac{d i_{L}}{d t} & =-u v_{C_{1}}+V_{i n} \\
C \frac{d v_{C_{1}}}{d t} & =u i_{L}-\frac{v_{C_{1}}}{R}+\frac{V_{2}}{R}
\end{aligned}
$$

The control law, $u$ can only take two values $u \in\{0,1\}$. Nevertheless, as usual, we consider its average value and, thus, $u$ is a continuous variable $u \in[0,1]$.

\section{B. Normalized Model}

In order to simplify the study, system (8)-(9) is normalized by using the following change of variables

$$
\begin{aligned}
x_{1} & =\frac{1}{V_{i n}} \sqrt{\frac{L}{C}} i_{L} \\
x_{2} & =\frac{v_{C_{1}}}{V_{i n}} \\
z_{2} & =\frac{V_{2}}{V_{i n}}
\end{aligned}
$$

and defining

$$
\tilde{t}=\omega t
$$


as a new time variable with

$$
\omega_{0}=\frac{1}{\sqrt{L C}}
$$

yielding

$$
\begin{aligned}
& \dot{x}_{1}=-u x_{2}+1 \\
& \dot{x}_{2}=u x_{1}-a x_{2}+a z_{2}
\end{aligned}
$$

where $a=\frac{1}{R} \sqrt{\frac{L}{C}}$.

Notice that eliminating $u$ in (15)-(16) we obtain

$$
x_{1}\left(1-\dot{x}_{1}\right)=x_{2}\left(\dot{x}_{2}+a x_{2}-a z_{2}\right) .
$$

This relationship will be used later.

\section{Controller Design}

System (15)-(16) is not directly matchable with system (3)-(4). The main problem is that two pairs of equations have to be matched while only one degree of freedom $u$ is available. Another change of variables is needed in order to get a model structure similar to (3)-(4). For this, define

$$
\begin{aligned}
& y_{1}=\frac{x_{1}^{2}+x_{2}^{2}}{2} \\
& y_{2}=x_{1}-a x_{2}^{2}+a x_{2} z_{2}+y_{20}
\end{aligned}
$$

where $y_{20}$ is an offset term that will be a tuning parameter. From (18)-(19), it is easy to see that

$$
\begin{aligned}
\dot{y}_{1}= & y_{2}-y_{20} \\
\dot{y}_{2}= & 1+2 a^{2} x_{2}^{2}-3 a^{2} z_{2} x_{2}+a^{2} z_{2}^{2}+a x_{2} \dot{z}_{2} \\
& -u\left(x_{2}+2 a x_{1} x_{2}-a z_{2} x_{1}\right)
\end{aligned}
$$

It is not easy to obtain a simple relationship $x_{1}=$ $f\left(y_{1}, y_{2}\right)$ and $x_{2}=f\left(y_{1}, y_{2}\right)$ due to complexity of definitions (18)-(19).

Looking at the target system structure (3)-(4) and comparing it with $(20)-(21)$ the choice $k_{a_{1}}=0$ is obvious resulting, as target system

$$
\begin{aligned}
& \dot{y}_{1}=y_{2}-y_{20} \\
& \dot{y}_{2}=-\omega^{2}\left(y_{1}-y_{10}\right)-k \Gamma\left(y_{2}-y_{20}\right)
\end{aligned}
$$

where, for simplicity, $k_{a_{2}}$ has been renamed to $k$.

The control law $u$ that matches (20)-(21) and (22)-(23) is

$$
\begin{aligned}
u= & \frac{1+2 a^{2} x_{2}^{2}-3 a^{2} z_{2} x_{2}+a^{2} z_{2}^{2}+a x_{2} \dot{z_{2}}}{x_{2}+2 a x_{1} x_{2}-a z_{2} x_{1}} \\
& +\frac{k \Gamma\left(y_{2}-y_{20}\right)+\omega^{2}\left(y_{1}-y_{10}\right)}{x_{2}+2 a x_{1} x_{2}-a z_{2} x_{1}}
\end{aligned}
$$

The ellipse parameters have to be defined as a function of the desired behavior. For this, it is necessary to obtain an analytical expression of the desired objective curve in the plane $x_{1}, x_{2}$. Assume that the desired time evolution for $x_{2}$ is

$$
x_{2}^{*}=A \sin \omega t+B
$$

where $A, B$ and $\omega$ take pre-specified values to obtain the desired evolution for $v_{C}$ and $i_{L}$ using (10)-(12), (13) and
(14). Time shift need not to be taken into account. Assume that the desired steady state for $x_{1}$ can be approximated by

$$
x_{1}^{*}=a \alpha_{0}+\alpha_{1} \cos \omega t+\beta_{1} \sin \omega
$$

Substituting (25) and (26) in (17), neglecting the second order harmonics and equating the bias, sin $\omega t$ and $\cos \omega t$ the parameters $\alpha_{0}, \alpha_{1}$ and $\beta_{1}$ can be obtained. The expressions appear in [5] and are omitted here for brevity.

The only question that remains now is to show that the desired behavior for $y_{1}$ and $y_{2}$ is an ellipse and to define the ellipse parameters $\left(\omega, y_{10}, y_{20}\right.$ and $\left.\mu\right)$ in terms of the desired behavior for $x_{2}$. For this, we have to obtain the desired evolution for $y_{1}$ and $y_{2}$ applying the change of variables (18)-(19) to (25) and (26).

$$
\begin{aligned}
y_{1}= & \frac{1}{2}\left[\left(a \alpha_{0}+\alpha_{1} \sin \omega t+\beta_{1} \cos \omega t\right)^{2}\right. \\
& \left.+\left(A_{1} \sin \omega t+B_{1}\right)^{2}\right] \\
y_{2}= & a \alpha_{0}+\alpha_{1} \sin \omega t+\beta_{1} \cos \omega t-a\left(A_{1} \sin \omega t+B_{1}\right)^{2} \\
& +a\left(A_{1} \sin \omega t+B_{1}\right) z_{2}+y_{20}
\end{aligned}
$$

Expanding these expressions in Fourier terms yield

$$
\begin{aligned}
y_{1}= & y_{1}^{(0)}+y_{1}^{(11)} \cos \omega t+y_{1}^{(12)} \sin \omega t+y_{1}^{(21)} \cos 2 \omega t \\
& +y_{1}^{(22)} \sin 2 \omega t \\
y_{2}= & y_{2}^{(0)}+y_{2}^{(11)} \cos \omega t+y_{2}^{(12)} \sin \omega t+y_{2}^{(21)} \cos 2 \omega t \\
& +y_{2}^{(22)} \sin 2 \omega t
\end{aligned}
$$

Equating the terms an expression for the Fourier coefficients can be obtained. The expressions appear in [5]. Assuming that the double frequency terms, $y_{1}^{(21)}, y_{1}^{(22)}$, $y_{2}^{(21)}, y_{2}^{(22)}$ can be neglected, these expressions can be approximated by an ellipse in the plane $y_{1}, y_{2}$ since, using the expressions previously obtained for $\alpha_{0}, \alpha_{1}$ and $\beta_{1}$,

$$
\begin{aligned}
& \omega y_{1}^{(11)}=-y_{2}^{(12)} \\
& \omega y_{1}^{(12)}=y_{2}^{(11)}
\end{aligned}
$$

The parameters of this ellipse are given by

$$
\begin{aligned}
y_{10} & =y_{1}^{(0)} \\
y_{20} & =y_{2}^{(0)} \\
\mu & =\omega^{2}\left(\left(y_{1}^{(11)}\right)^{2}+\left(y_{1}^{(12)}\right)^{2}\right)
\end{aligned}
$$

\section{Control Law for the Full Converter}

In this section the previous control law is extrapolated to consider that the boost inverter is composed of two dcdc converters and, thus, it has two control signals. The model of the system is

$$
\begin{aligned}
L_{1} \frac{d i_{L_{1}}}{d t} & =-u_{1} v_{C_{1}}+V_{i n} \\
C_{1} \frac{d v_{c_{1}}}{d t} & =u_{1} i_{L_{1}}-\frac{v_{C_{1}}}{R}+\frac{v_{C_{2}}}{R} \\
L_{2} \frac{d i_{L_{2}}}{d t} & =-u_{2} v_{C_{2}}+V_{i n} \\
C_{2} \frac{d v_{c_{2}}}{d t} & =u_{2} i_{L_{2}}-\frac{v_{C_{1}}}{R}+\frac{v_{C_{2}}}{R}
\end{aligned}
$$


By comparison of systems (36)-(39) and (8)-(9) we can notice a similar structure for the pairs of current and voltage of both boost dc-dc converters. Therefore, the two control laws are easy to be obtained. The first control law $u_{1}$ is almost the same expression as (24), the only difference is that $z_{2}$ must be replaced by $x_{4}$. Control law $u_{2}$ is obtained by symmetry. The control laws are

$$
\begin{aligned}
u_{1}= & \frac{1+2 a_{1}^{2} x_{2}^{2}-3 a_{1}^{2} x_{2} x_{4}+a_{1}^{2} x_{4}^{2}+a_{1}^{2} x_{2} \dot{x}_{4}}{x_{2}+2 a_{1} x_{1} x_{2}-a_{1} x_{4} x_{1}} \\
& +\frac{k_{1} \Gamma_{1}\left(y_{2}-y_{20}\right)+\omega^{2}\left(y_{1}-y_{10}\right)}{x_{2}+2 a_{1} x_{1} x_{2}-a_{1} x_{4} x_{1}} \\
u_{2}= & \frac{1+2 a_{2}^{2} x_{4}^{2}-3 a_{2}^{2} x_{2} x_{4}+a_{2}^{2} x_{2}^{2}-a_{2}^{2} x_{4} \dot{x}_{2}}{x_{4}-2 a_{2} x_{3} x_{4}+a_{2} x_{2} x_{3}} \\
& +\frac{-k_{2} \Gamma_{2}\left(y_{4}-y_{40}\right)+\omega^{2}\left(y_{3}-y_{30}\right)}{x_{4}-2 a_{2} x_{3} x_{4}+a_{2} x_{2} x_{3}}
\end{aligned}
$$

The expressions for derivatives $\dot{x}_{2}$ and $\dot{x}_{4}$ are taken directly from the normalized equations of the boost inverter.

\section{E. Simulation Results}

The following simulations are made considering $V_{\text {in }}=$ $10 \mathrm{~V}, R=100 \Omega, L_{1}=100 \mu \mathrm{H}, L_{2}=100 \mu \mathrm{H}, C_{1}=100 \mu \mathrm{F}$, $C_{2}=100 \mu \mathrm{F}$. The desired frequency and voltage amplitude are $50 \mathrm{~Hz}$ and $40 \mathrm{~V}$ respectively.

In this case, as the dc-dc converter voltages must have a phase shift of $180^{\circ}$ the normalized desired voltages are

$$
\begin{aligned}
& x_{2}^{*}=A \sin \omega t+B \\
& x_{4}^{*}=-A \sin \omega t+B
\end{aligned}
$$

Parameter $A$ has to be the half of the desired output voltage amplitude and $B$ is chosen, so that, $x_{2}$ and $x_{4}$ are always positive. In order to obtain this voltage, the parameters are $A=2$ and $B=10$ with $\omega=0.0314$ in the normalized variables $x_{1}, x_{2}$.

The ellipse parameters, using the previous development, result $y_{10}=51.14, y_{30}=51.14, \mu_{1}=\mu_{2}=0.395$. On the other hand, the tuning parameters are chosen as $k_{1}=k_{2}=$ 0.8 and $y_{20}=0, y_{40}=0$.

Figure 6 shows the results of a simulation using a sample time of 0.001s. Both dc-dc converters achieve the desired limit cycle.

Figure 7 shows the boost inverter input and output voltages. In this figure only the steady state behavior is shown. It can be seen that the desired amplitude is not achived. Indeed, the previous design does not impose that the phase shift between signals $v_{c_{1}}$ and $v_{c_{2}}$ is $180^{\circ}$. Figure 8 shows that this requirement is not achieved. Next Section will deal with this problem.

\section{Voltage Synchronization}

In the previous design, we found that the voltage signal did not present a phase shift of $180^{\circ}$. In order to get the desired output voltage, we need to synchronize these signals. In this section, a PLL is added to achieve the desired phase shift.

A PLL is a device which causes one signal to track another one. This tracking is in frequency as well as in phase.
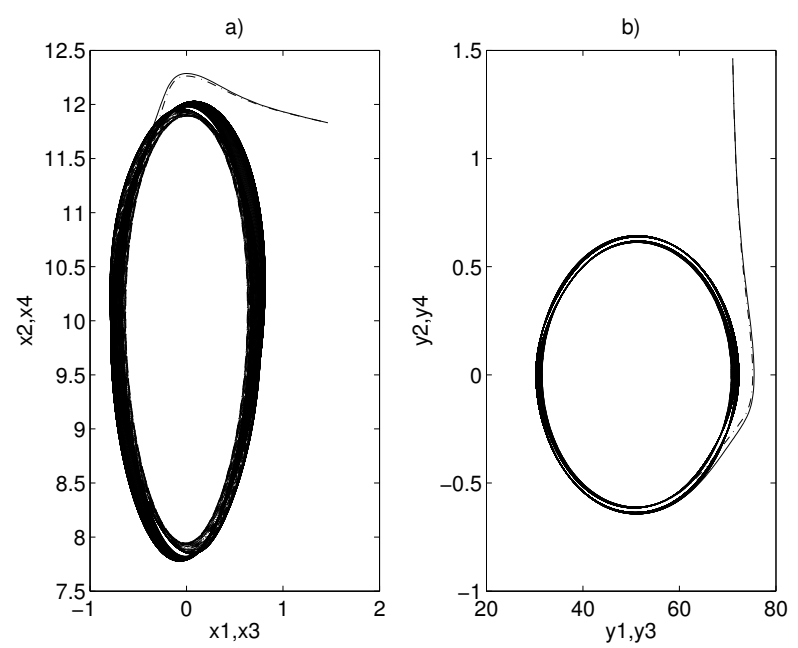

Fig. 6. a) State spaces $\left(x_{1}, x_{2}\right)$ (solid) and $\left(x_{3}, x_{4}\right)$ (dashed); b) state spaces $\left(y_{1}, y_{2}\right)$ (solid) and $\left(y_{3}, y_{4}\right)$ (dashed).

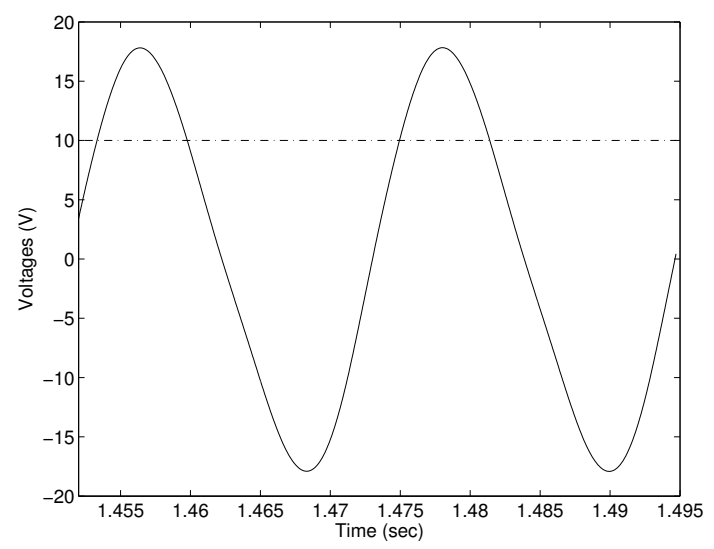

Fig. 7. Output (solid) and input (dashed) voltages of the boost inverter.

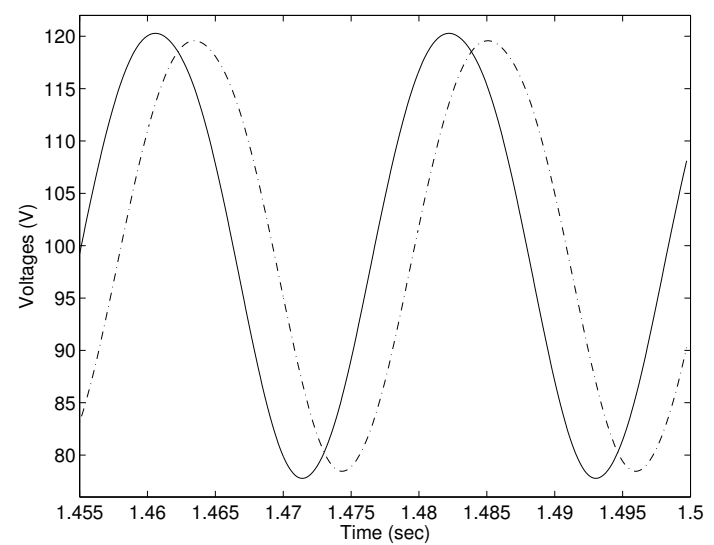

Fig. 8. Output voltages of the first (solid) and second (dashed) boost dc-dc converters. 
A PLL consists basically of three parts [7]-[8]: a phase detector (PD), a loop filter (LF) and a voltage-controlled oscillator (VCO). A functional scheme of the device is shown in Fig. 9. Signal $x_{i}$ is the reference signal and $x_{0}$ is the synchronized signal. The PD is a signal multiplier and it has a gain $k_{d}$. The resultant product is filtered in the LF, so that, the higher frequency component is eliminated. The $\mathrm{VCO}$ is a frequency-modulated oscillator, and it generates a signal which is in phase quadrature with respecto to the input signal.

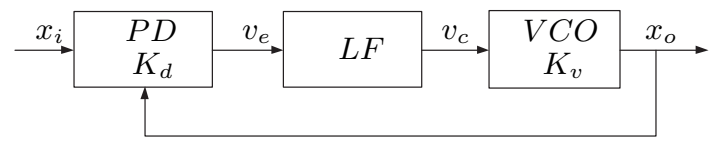

Fig. 9. Basic PLL block diagram.

\section{A. PLL Applied to the Boost DC-AC Converter}

The objective is to synchronize the voltage curves with a phase shift equal to $180^{\circ}$. The scheme of Fig. 10 is used. The voltage of the second dc-dc converter, $x_{4}$, is taken as reference signal and the voltage of the first dc$\mathrm{dc}$ converter, $x_{2}$, is the signal to be synchronized. The first dc-dc converter with its controller can be regarded as a VCO: the controller receives as input a frequency increment that it is ordered by the PLL. This frequency increment is added to the nominal frequency, and the resultant frequency is entered in (40). The output of the converter is a sinusoidal signal of that frequency.

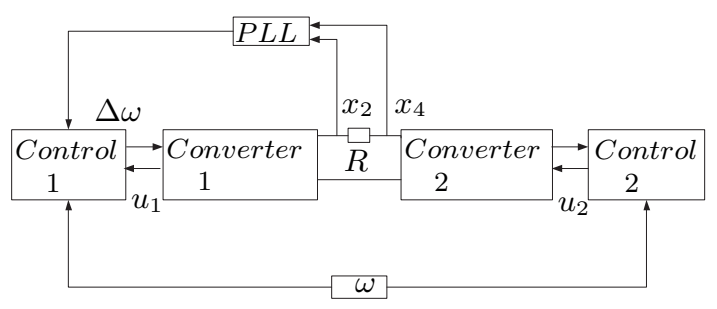

Fig. 10. Block diagram of boost dc-ac converter with output voltages synchronized by PLL.

The PLL block diagram appears in Fig. 11. The multiplier obtains the product $x_{2}^{*} \times x_{4}^{*}$ in such a way that, see [7], its output, once filtered by a low pass filter (LPF), is a measure of the deviation of the phase shift with respect to $90^{\circ}$. For this reason, one of the inputs of the multiplier, e.g. $x_{2}^{*}$, is obtained passing the voltage $x_{2}$ through a high pass filter (HPF) in order to eliminate its continuous component. On the other hand, $x_{4}^{*}$ is obtained after passing $x_{4}$ through another HPF and, then, changing its sign and integrating. In this way if $x_{4}=B_{2}+A_{2} \sin (\omega t)$, then, $x_{4}^{*}=-\frac{A_{2}}{\omega} \sin \left(\omega t-\frac{\pi}{2}\right)+C$. Constant $C$ is eliminated by the LPF.

1) Simulation Results: The high pass filter applied are: $\frac{1.4 s}{s+\omega}$ for the reference signal and $\frac{1.4 s}{s+\omega+\Delta \omega}$ for the signal to be synchronized.

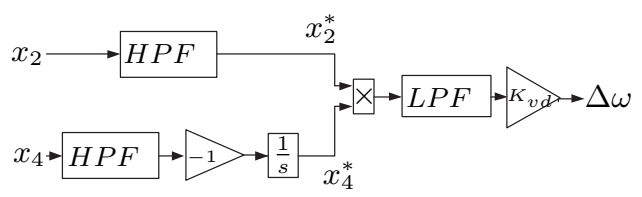

Fig. 11. Conceptual block diagram of PLL applied to boost dc-ac converter.

The LPF is a second order Butterworth filter [9]:

$$
\frac{64 \cdot 10^{-} 6\left(\frac{\sqrt{2}}{2}-\frac{\sqrt{2}}{2} j\right)\left(\frac{\sqrt{2}}{2}+\frac{\sqrt{2}}{2} j\right)}{\left(s+0.008\left(\frac{\sqrt{2}}{2}-\frac{\sqrt{2}}{2} j\right)\right)\left(s+0.008\left(\frac{\sqrt{2}}{2}+\frac{\sqrt{2}}{2} j\right)\right)}
$$

The value of the PLL gain is $K_{d v}=5 \cdot 10^{4}$.

The results of the PLL application are shown in Fig. 12. The voltages $v_{c_{1}}$ and $v_{c_{2}}$ present a phase shift of $180^{\circ}$. In Fig. 13 the boost inverter output voltage is represented. This voltage signal is compared with the ideal boost inverter output, getting a excellent result.

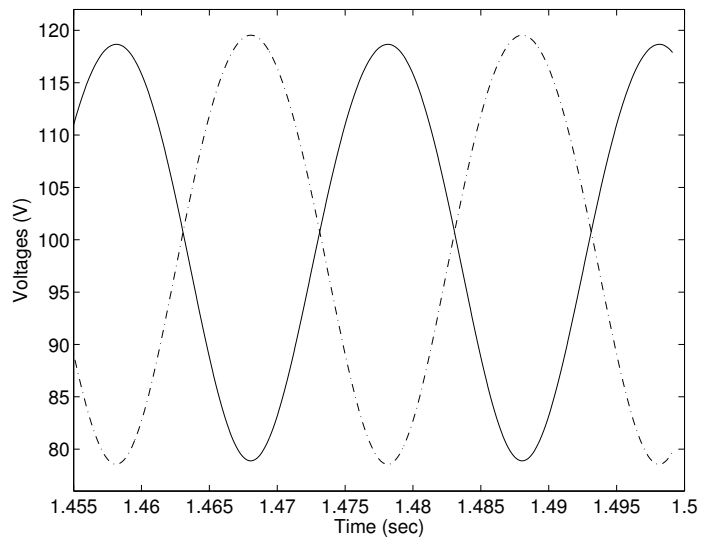

Fig. 12. Output voltage of the first (solid) and second (dashed) boost dc-dc converters.

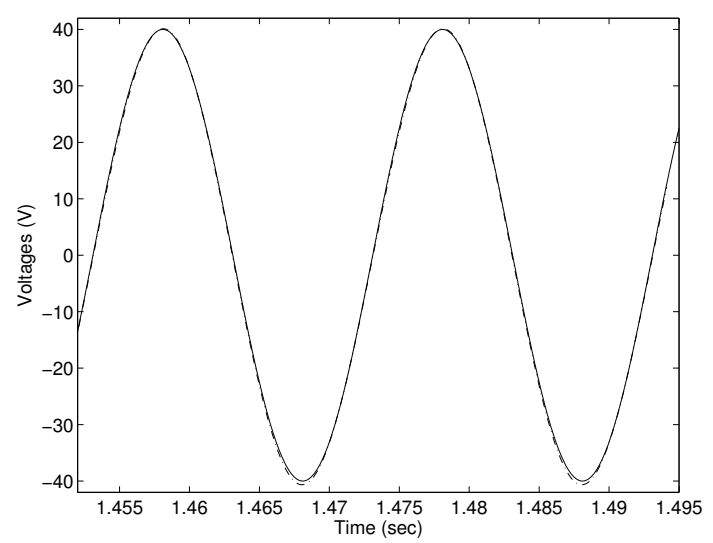

Fig. 13. Ideal (solid) and simulated (dashed) output voltages with PLL.

\section{B. Synchronizing with the Electrical Network}

In order to synchronize the boost inverter output signal with the electrical network, the voltage signals of both dcdc converters $\left(v_{c_{1}}, v_{c_{2}}\right)$ are treated with a PLL to get 
the aim (Fig. 14). Voltage $v_{c_{1}}$ is synchronized with the network voltage without phase shift, while voltage $v_{c_{2}}$ is synchronized with the network voltage with a phase shift of $180^{\circ}$.

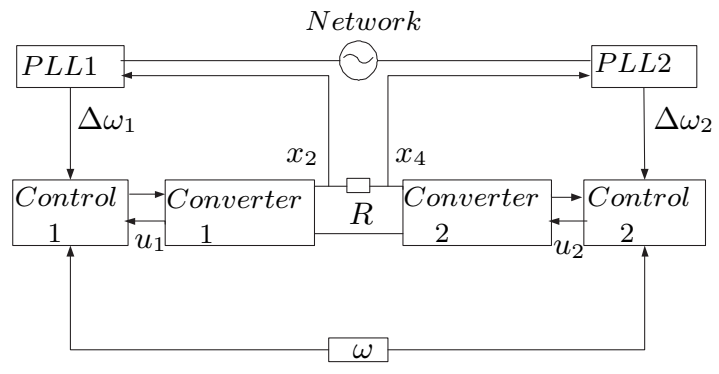

Fig. 14. Conceptual block diagram of PLL applied to the boost dc-ac converter for network synchronization.

1) Simulation Results: The network voltage is

$$
V_{\text {net }}=325 \sin (100 \pi t) \text {. }
$$

The filter parameters and gain used in the PLLs are the same which were used previously.

The performance of the synchronization of the boost dc-ac converter with the electrical supply voltage is represented in Fig.15.

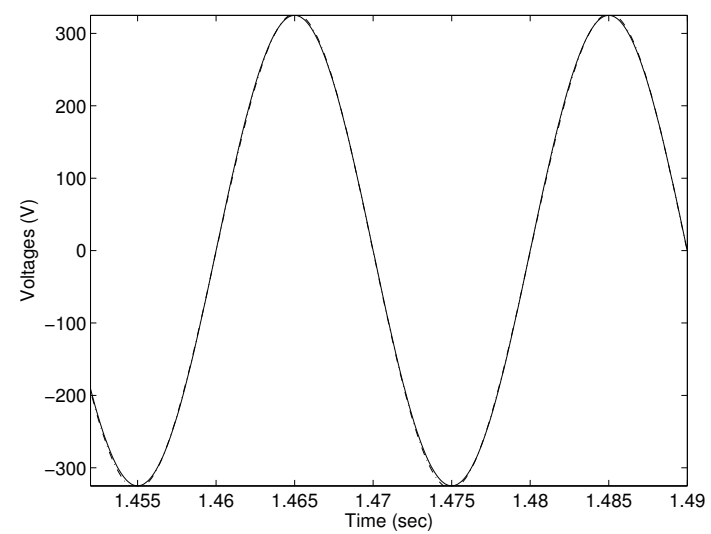

Fig. 15. Electrical supply voltage (solid) and simulated output voltage synchronized with PLLs (dashed).

\section{Conclusions}

This paper presents a control strategy for the boost dc-ac converter. The method is based on energy shaping and generation of a limit cycle and does not need the introduction of time-dependent reference signals. It has been shown that the resultant controller achieves the objective provided that a phase locked loop is added. The same idea has been used in order to solve the problem of network synchronization.

Open problems are the consideration of unknown and non-resistive loads.

\section{Acknowledgments}

This research has been supported by the MCyT-FEDER grant DPI2003-00429.

\section{REFERENCES}

[1] J. Aracil, F. Gordillo, and J. Acosta, "Stabilization of oscillations in the inverted pendulum," in XV IFAC World Congress, 2002.

[2] F. Gómez-Estern, J. Aracil, and F. Gordillo, "The Hopf bifurcation and controlled oscillations in electromechanical systems," in Proceedings of the MED'02, Lisbon, Portugal, 2002.

[3] F. Gordillo, J. Aracil, and F. Gómez-Estern, "Stabilization of autonomous oscillations and the Hopf bifurcation in the ball and beam," in Proceedings of the $C D C^{\prime} 02$, vol. 4, Las Vegas, USA, 2002, pp. 3924-3925.

[4] J. Aracil and F. Gordillo, "On the control of oscillations in DCAC converters," in Proc. IECON'02, Sevilla, Spain, 2002.

[5] F. Gordillo, D. Pagano, and J. Aracil, "Autonomous oscillation generation in electronic converters," in Proceedings of the 2004 International Workshop on Electronics and System Analysis, IWESA'04, 2004, pp. 57-65.

[6] R. O. Cáceres and I. Barbi, "A boost DC-AC converter: analysis, design, and experimentation," in IEEE Transactions on Power Electronics, vol. 14, 1999, pp. 134-141.

[7] G.-C. Hsieh and J. C. Hung, "Phase-Locked Loop techniques-a survey," in IEEE Transactions on Industrial Electronics, vol. 1, 1996, pp. 609-615.

[8] D. Abramovich, "Phase-Locked Loop: a control centric tutorial," in Proceedings of the American Control Conference, vol. 1, Anchorage, AK, 2002, pp. 1-15.

[9] Ifeachor, E. c., and W. J. Barrie, Digital signal processing: a practical aproach. Addison-Wesley, 1993. 